\title{
Principle and Control Technology of Automobile Braking Noise
}

\author{
Yuanpeng Zhu
}

\author{
Wuhan University of Technology, Wuhan 430070, China \\ whutyuanpeng@whut.edu.cn
}

\begin{abstract}
With the increasing demands of car ride comfort, automobile brake noise has become a serious concern, so reducing automobile brake noise has become the primary task of automobile manufacturers and parts suppliers. This paper briefly describes the classification of automobile brake noise, the mechanism, and the method of controlling the braking noise of automobile.
\end{abstract}

Keywords: automobile; brake noise; control method; mechanism

For the brake of the car, the phenomenon of vibration and noise due to friction has long existed, and usually occurs in a certain frequency range, and with the braking system stiffness, braking speed, braking pressure and a variety of environmental conditions closely related. Brake noise frequency is usually more single, but occasionally appears at the same time lower harmonic. Different standards have different classification for brake noise, but they use the frequency of noise as the characteristic parameter of classification.

\section{Classification and generation of braking noise}

Braking noise frequency range from tens of hertz to tens of hertz, according to the frequency of different, roughly can be divided into three categories. The braking noise is mainly determined by the stability of the braking system. The radial coupling of the friction lining changes the natural frequency and mode of the system, but does not change the stability of the system, and the friction coupling will change the system stability. Brake noise generation, whether high or low frequency, the mechanism is the same.

\subsection{Low frequency noise}

The noise from the start of the brake to the stop is generally low frequency noise, usually between 100 and $1000 \mathrm{~Hz}$. The friction between the brake disc and the surface of the friction material causes the brake and chassis to vibrate. To reduce the low-frequency noise, adjust the structure does not work, mainly by improving the friction material, friction plate shape and surface to achieve. When the friction coefficient and velocity curve of the pad and the disk show the friction slip / negative slope friction coefficient as shown in Fig. 1, the liner, the disc and the caliper will produce low frequency vibration and low frequency noise. Come, as shown in Fig. 2. 


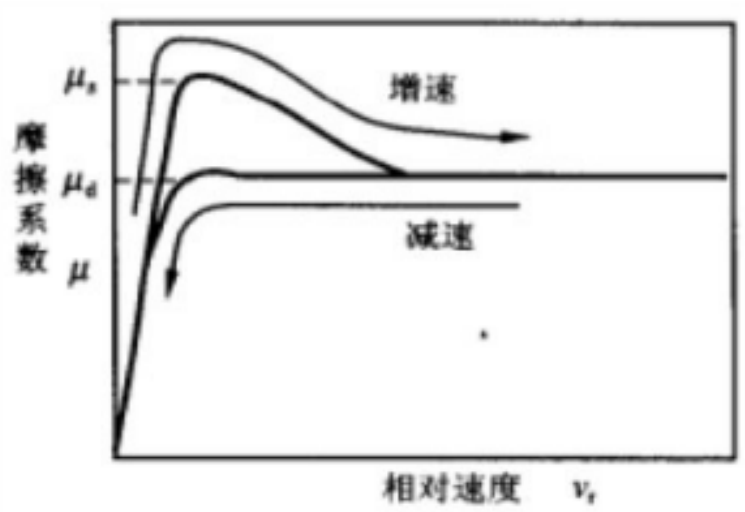

Fig.1 Coefficient of friction of pad and disk body - Relative velocity curve

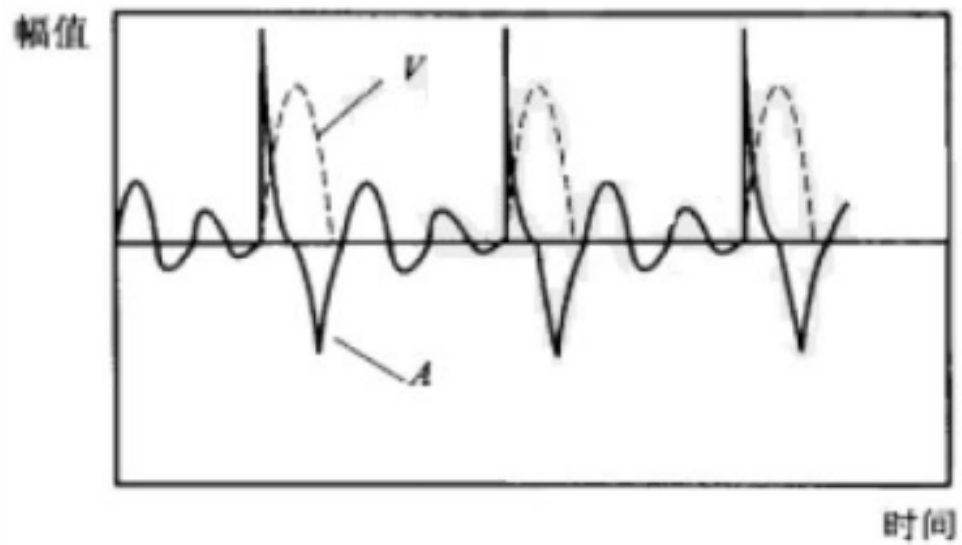

Fi.2 Low-frequency vibration of the liner, disc and caliper

A-brake caliper acceleration; V-liner plate relative speed

\subsection{Low frequency screaming noise}

The screams in the braking process are low frequency screams at frequencies from 1 to $6 \mathrm{~Hz}$, mainly due to friction that causes the coupling between the parts of the brake. In particular, when the parts are moved at different speeds at different frequencies, the frictional force causes the modal coupling to stimulate a strong vibration to produce noise. Adjusting the structure, especially the isolation of the brake disc body to the caliper mode, is particularly effective for reducing low frequency noise.

\subsection{High frequency screaming noise}

The noise that occurs during parking is a high frequency scream at frequencies above $6 \mathrm{kHz}$ due to the self-excited vibration caused by the elastic vibration of the friction pad. High frequency screaming up to about $120 \mathrm{~dB}$, is an unbearable scream, the human body has a great harm, but also one of the important sources of noise in urban noise. High-frequency screaming noise is often related to the circumferential mode of the brake disc body, but also with the stiffness of the pad and disk body, increasing the dynamic stiffness of the disk can reduce the occurrence of high frequency screaming noise. 


\subsection{Influencing factors}

Some of the direct factors that affect the braking noise of the vehicle are: structural characteristics such as the quality of the block, the disc and the caliper, the stiffness, the damping and the geometric dimensions; the material performance parameters such as the friction coefficient, the elastic modulus and the hardness; Conditions such as brake pressure and braking speed; braking environments such as temperature and humidity, they affect the noise by friction coefficient. In addition, due to the complexity of its influencing factors, despite the academic research brake noise has been a long time, but there are still many problems have not been resolved. At the same time, this topic has attracted many scholars, including tribology, mechanics, materials science and even computer science and other fields of interest, and published a lot of research results.

\section{Research status of automobile braking noise}

In fact, as early as the 1930s on the brake noise on the study, the researchers have been resolved to solve specific problems of practical application methods and analysis of the various models, but until now, the mechanism of the problem and solve the problem Methods have not yet reached a consistent conclusion because it involves not only the complex dynamics of the brake system, but also related to the braking conditions and practical environment, which increased the difficulty of the study carried out.

With the development of control technology and the reduction of cost, the application of new materials, the continuous improvement of test technology, the development of computer software, the development of automobile noise technology is very rapid. CAE software development has made the car noise can be used to predict the computer model. However, due to theoretical research requires a certain premise environment to derive, and the actual working environment is quite complex, most can only qualitative description of the problem, from the actual situation. Therefore, the current research on the automobile brake noise is mainly based on the experimental method.

However, the limitations of studying only by experimentation are obvious, for example, some system parameters are not easy to change, and because of the different experimental conditions, the results will change, the conclusion of the verifiability is relatively poor. In addition, the results obtained through the experiment are often based on the experience of the summary, for the sudden situation and experience outside the situation is difficult to deal with. At present, the brake screaming noise model established by the finite element method is a relatively perfect model to date, but the finite element method is used to study the obtained results and the accuracy is not high.

\section{Method of reducing automobile braking noise}

The purpose of the study of brake noise is to design the vehicle stage, through simulation and other measures to minimize the brake noise. Therefore, it is necessary to use the brakes as a whole for finite element analysis. On the basis of modal analysis and modal modeling, the parameters such as structural parameters and material parameters of the 
brake are proposed to reduce the friction induced by friction. Generally speaking, the method of reducing the noise of automobile braking is mainly from the aspects of design and manufacture and usage habits.

\subsection{From the design and manufacturing perspective}

If the drum brakes are to increase the rigidity of the brake drum and reduce the rigidity of the brake shoe. As the natural frequency of the brake drum increases, the braking noise gradually decreases. Therefore, the design of the brake, you can improve the rigidity of the brake drum, in order to improve its natural frequency, but no need to infinitely improve the rigidity, when the braking noise close to the background noise, enough to meet the requirements. The reduction of the rigidity of the brake shoe can reduce the natural frequency of the vibration and improve the pressure distribution and contact between the friction lining and the drum to reduce the noise. In addition, the damping action of the structural parts of the brake can be used to attenuate the energy and reduce the effect of the noise. For disc brakes, the way to reduce the brake noise is basically the same, but also mainly relies on the rational design of parts, to limit vibration, to take damping measures and other methods. However, some methods have to resort to remedial measures to ensure the braking effect.

\subsection{From the use of angle}

During the use of the car, it is difficult to reduce and eliminate the braking noise by changing the structure of the brake, and can only prevent and eliminate the braking noise through the correct driving operation and timely maintenance repair. Braking, if there is a very sharp braking noise, can be excluded by the following method.

An anti-noise piece is added between the piston and the brake lining of the brake assembly and an inclination is formed on the piston to ensure that the brake pad and the brake disc are in flexible contact during braking so that the brake pad there is no abnormal noise in normal wear. Braking, if the brake pads to move to the side of the impact sound, you can use solder plating method to eliminate the brake pad and clamp between the excessive gaps. It should be noted that the solder should be coated on the opposite side of the direction of travel, to prevent the role of braking force in the failure.

\section{Conclusion}

So far, the research of brake noise has been close to 100 years, and has made great progress in this field. But because of the complexity of the problem in this field, there are still a large number of blank theory and technology, there are many problems still haunt the domestic and foreign researchers, this means that the relative to other research direction of automobile technology, the vehicle noise control in the field has not yet formed by developed countries technological monopoly pattern. Therefore, it is of great significance to carry out the research on the theory of brake noise and engineering application, and to seize the highest point of technology. 


\section{References}

[1] X.F. Guan, W.H. Liu. Optimization of Automobile Braking Noise[J]. Automotive Engineer.2015 (04)

[2] B.W. Liu, Y. Yang, X. Xiong. Study on Braking Noise of Automobile [J].Tribology Journal of Tribology. 2009 (04)

[3] Z.G. Hai, H. Gong, J.P. Shi, H. Liu. Analysis of braking noise improvement method[J]. Automotive Technology.2015 (09).

[4]. X.H. Yu; G. Yu. Mechanism and control method of automobile brake noise [J]. Equipment manufacturing technology.2009 (06) 